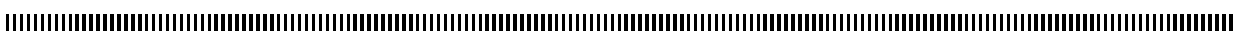

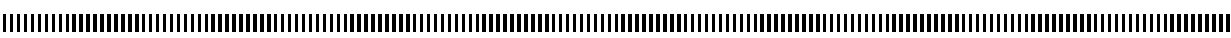
| |

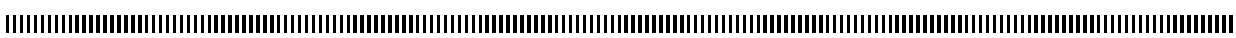

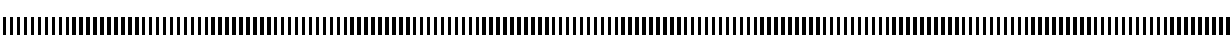
| | |

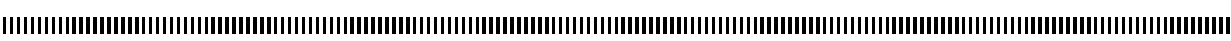

\title{
AN ERROR ESTIMATE IN IMAGE PROCESSING
}

\author{
Philippe Destuynder * _ Mohamed Jaoua ${ }^{* *}$ — Hela Sellami*** \\ * Département de mathématiques \\ Conservatoire National des Arts et Métiers \\ 292 rue Saint Martin, 75003 Paris \\ FRANCE \\ destuynd@cnam.fr \\ ** Université Française d'Egypte, PO Box 21, Chorouq, Cairo, Egypt \\ $\&$ \\ Laboratoire J.-A. Dieudonné \\ Université de Nice-Sophia Antipolis, \\ UMR 6621 du CNRS, Parc Valrose 06108 Nice cedex 2 \\ France \\ jaoua@polytech.unice.fr \\ *** LAMSIN-ENIT \\ Ecole Nationale d'Ingénieurs de Tunis \\ BP 37, 1002 Tunis-Belvédère \\ TUNISIE \\ hela.sellami@lamsin.rnu.tn
}

|IIIIIIIIIIIIIIIIIIIIIIIIIIIIIIIIIIIIIIIIIIIIIIIIIIIIIIIIIIIIIIIIIIIIIIIIIIIIIIIIIIIIIIIIIIIIIIIIIIIIIIIIIIIIIIIIIIIIIIIIIIIIIIIIIIIIIIIIIIIIIIIIIIIIIIIIII

ABSTRACT. A new interpolation error estimate for a finite element method for image processing is proved in this paper. The suggested scheme is based on the Raviart-Thomas' one, extended to a non linear formulation. The numerical trials run confirm the accuracy of the restoration algorithm.

RÉSUMÉ. Une nouvelle estimation de l'erreur d'interpolation dans une méthode d'éléments finis utilisée en traitement d'images est prouvée dans cet article. L'élément proposé est celui de RaviartThomas, prolongé à une formulation non linéaire. Les tests numériques confirment la précision de la méthode.

KEYWORDS : Finite element method, interpolation, image processing, functional space BV.

MOTS-CLÉS : Méthode des éléments finis, interpolation, traitement d'images, espace BV.

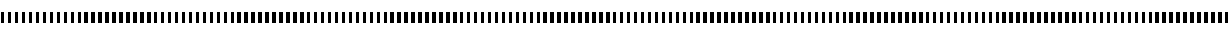

Received, February 24,2010 ,

ARIMA Journal, vol. 15 (2012), pp. 61-81

Last revised, November 18, 2011,

Accepted, May 19, 2012

| |

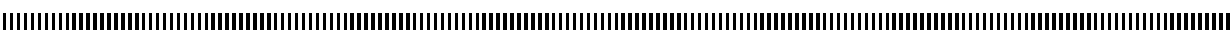

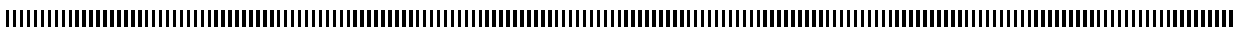

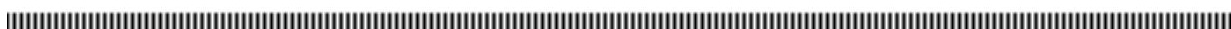




\section{Introduction}

Restoration is a crucial step in image processing. Numerous algorithms have been proposed recently to tackle the problems of noise removal and image restoration in real images $[18,7,3,6]$. Due to this large number of approaches, there is a need to compare and quantify the numerical results provided by each of them in order to make an idea.

In this paper, we shall be presenting a dual approach to solve the restoration variational problem introduced in [12], where the implemented algorithm of resolution requires a projection over a convex set. Preserving conformity of the approximation space by using a global projection on the whole mesh would be too costly, besides artificially propagating the local error. This is the reason why this aspect is analyzed and discussed hereafter. Additional interesting analyses can be found in several papers, especially those by G. Acosta and R. G. Duran [1, 13, 14, 15].

Section 2 of the present paper is devoted to recalling a smoothed total variation model proposed by several authors $[3,10]$, and to presenting the dual approach introduced in [12] to tackle the issue. In section 3, our interest focuses on the use of quadrilateral finite element methods used to discretize the domain and to estimate the interpolation error generated. Numerical esults and their discussions are provided in section 4, before we conclude in section 5 .

\section{A smoothed total variation model for image restoration}

Let us denote by $u$ an original, non-corrupted image, which is a function defined on some rectangle $\Omega=] 0, l[\times] 0, L[$, and let $f$ be noisy image defined by :

$$
f=u+\mu,
$$

where $\mu$ is a white additive Gaussian noise.

Reconstructing $u$ from $f$ by simply minimizing the quadratic misfit $\min _{v} \frac{1}{2} \int_{\Omega}|v-f|^{2}$ over the space one wishes the image to dwell in is an inverse problem which is well known to be severely ill-posed [16]. It is thus necessary to regularize this problem by adding a regularizing term bringing an a priori constraint on the solution or its derivatives. Following several authors ([3], [17]), we shall choose the regularization leading to the following optimization problem:

$$
\min _{v}\left\{J(v)=\frac{1}{2} \int_{\Omega}|v-f|^{2}+\varepsilon \int_{\Omega} \sqrt{1+\alpha|\nabla v|^{2}}\right\}
$$

\section{A R I M A}


where $\alpha$ and $\varepsilon$ are two parameters to be adjusted in order to get satisfactory results.

The reason of such a choice are the following :

- For (locally) small values of $|\nabla v|$, the following equivalence holds :

$$
\sqrt{1+\alpha|\nabla v|^{2}} \sim 1+\frac{\alpha}{2}|\nabla v|^{2},
$$

which makes problem (2) a classical filtering process, damping down the small perturbations of the gradient.

- For large values of $|\nabla v|$, it is on the other hand the following equivalence which holds:

$$
\sqrt{1+\alpha|\nabla v|^{2}} \sim \sqrt{\alpha}|\nabla v|
$$

which makes problem (2) alike the total variation problem proposed by Osher, Rudin and Fatemi [18], thus enabling the description of contours.

The minimization problem (2) is then expected to meet both goals of denoising and preserving discontinuities.

It is well known that the solution of this problem belongs to the space $B V(\Omega)$ of functions with bounded variations defined by ([18], [3]):

$$
B V(\Omega)=\left\{v \in L^{2}(\Omega) ; T V(v)<+\infty\right\}
$$

where the total variation $T V$ is defined by:

$$
T V(v):=\sup \left\{\int_{\Omega} v \operatorname{div} \varphi d x ; \varphi \in \mathcal{C}_{0}^{1}(\Omega) \text { and }|\varphi|_{L^{\infty}(\Omega)} \leq 1\right\} .
$$

The most valuable feature of that space is to allow discontinuities along line curves (in 2D). It is thus presumably well fit to describe contours. There is however a price to pay in the numerical approximation of this problem, which is quite a difficult task to go through since it needs to approximate the space $B V$.

Many authors have proposed methods to solve problem (2) among which one can refer to the primal approach proposed by Destuynder [11].

\subsection{The dual approach}

In seek of a simpler way to solve the problem (2), we shall use the duality theory by introducing the Lagrangian $\mathcal{L}(v, q, \mu)$ [9], defined by:

$$
\mathcal{L}(v, q, \mu)=\frac{1}{2} \int_{\Omega}|v-f|^{2}+\varepsilon \int_{\Omega} \sqrt{1+\alpha|q|^{2}}+\int_{\Omega}(\nabla v-q) \mu
$$


Therefore the solution of the dual problem is given by the extremality relation (see [12]):

$$
u=f+\sqrt{\alpha} \operatorname{div} \eta
$$

where $\eta$ is the Lagrange multiplier, solving the following dual problem :

$$
\min _{(\eta, z) \in \mathcal{D}}\left\{\mathcal{F}(\eta, z)=\frac{\alpha}{2} \int_{\Omega}|\operatorname{div} \eta|^{2}+\sqrt{\alpha} \int_{\Omega} f \operatorname{div} \eta+\frac{\beta}{2} \int_{\Omega}|\eta|^{2}-\int_{\Omega} z\right\}
$$

and $\beta$ is a small strictly positive regularization parameter.

The minimization of this problem is performed over the closed non empty convex set:

$$
\mathcal{D}=\left\{(\eta, z) \in H_{0}(\operatorname{div}, \Omega) \times L^{2}(\Omega) \mid \eta^{2}+z^{2} \leq \varepsilon^{2} ; z \geq 0\right\}
$$

where the Sobolev space $H_{0}(\operatorname{div}, \Omega)$ is defined by:

$$
H_{0}(\operatorname{div}, \Omega)=\left\{\eta \in\left[L^{2}(\Omega)\right]^{2}, \operatorname{div}(\eta) \in L^{2}(\Omega), \eta . \nu=0 \text { on } \Gamma\right\}, \text { with } \Gamma=\partial \Omega .
$$

Then by solving the dual problem (9), we get - for a small enough value of $\beta$ - an approximate value of the processed image by the optimality condition (8).

We are now interested in the approximation of the vector field $\eta$ in the space $H_{0}(\operatorname{div}, \Omega)$, in order to compute the processed image obtained by adding the corrector div $\eta$ to the noisy image $f$. For this purpose, we shall use the simplest first order Raviart-Thomas $H(\operatorname{div}, \Omega)$ quadrilateral finite element.

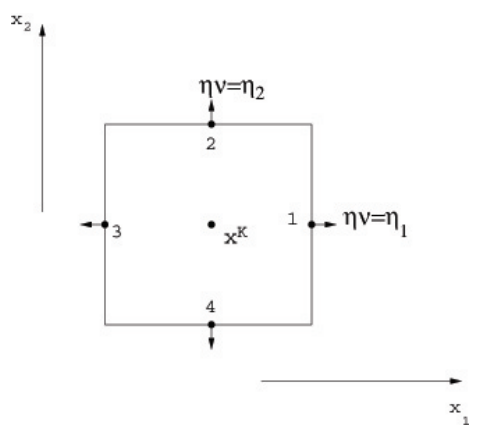

Figure 1. The Raviart-Thomas $H($ div, $\Omega)$ first order finite element

The relevant degrees of freedom are the normal fluxes of $\eta$ at the midpoints of the mesh edges, which are constant along the edges, since their continuity from one element

\section{A R I M A}


to the next one ensures the divergence of $\eta$ to remain in $L^{2}(\Omega)$.

In order to solve problem (9), one can use the steepest descent algorithm with projection (gradient with projection). Let us denote by $\Pi_{\mathcal{D}}$ the projection operator on $\mathcal{D}$ (see figure 2), then the algorithm writes down as follows:

The steepest descent algorithm with projection:

First guess: Choose $\left(\eta_{0}, z_{0}\right) \in \mathcal{D}$

$k$-th iteration: $\left(\eta_{k+1}, z_{k+1}\right)=\Pi_{\mathcal{D}}\left(\left(\eta_{k}, z_{k}\right)-\rho_{k} \nabla \mathcal{F}_{\beta}\left(\eta_{k}, z_{k}\right)\right)$

Stopping criterion: $\left\|\eta_{k+1}-\eta_{k}\right\|_{\left(L^{\infty}\right)^{2}} \leq \delta$,

where $\delta$ is some prescribed threshold

where $\rho_{k}$ is the $k$-th iteration optimal step.

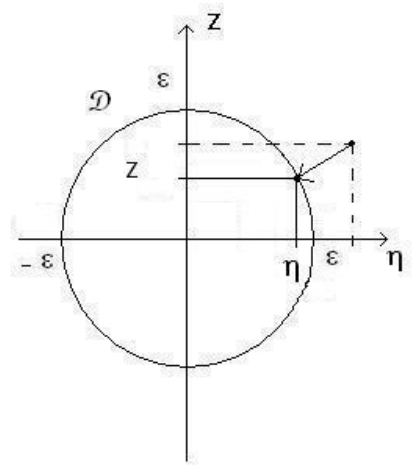

Figure 2. Projection over the convex $\mathcal{D}$.

Performing a conforming projection on the convex set $\mathcal{D}$ would make it a global operator and thus a somewhat complicated one to implement, since the degrees of freedom are connected from one element to the next one. This is the reason why local approximate projections, such as the projection on the center of the each element $K$, or the one on the interior edges midpoints, are preferred. We however need to choose the approximate projection operator providing the best error estimate. To that end, the interpolation error (related to $\eta$ ) needs to be estimated. Hopefully, it might enable us to preserve the order of approximation even though the local projection is non conforming with respect to the space $H(\operatorname{div}, \Omega)$. Before tackling that task, let us recall the variational formulation of the 
66 A R I M A - Volume 15-2012

dual problem.

\subsection{Variational formulation of the dual problem}

Let us consider the simplified problem obtained by assuming $z$ to be a given function taking its values in $[0, \varepsilon[$. Then, the solution $\eta$ of (9) turns out to solve the following problem:

$$
\min _{\eta \in K_{z}} \frac{\alpha}{2} \int_{\Omega}|\operatorname{div} \eta|^{2}+\sqrt{\alpha} \int_{\Omega} f \operatorname{div} \eta+\frac{\beta}{2} \int_{\Omega}|\eta|^{2},
$$

where $K_{z}$ is the closed convex set defined by:

$$
K_{z}=\left\{\eta \in H_{0}(\operatorname{div}, \Omega),|\eta| \leq \sqrt{\varepsilon^{2}-z^{2}}\right\} .
$$

and where $|\eta|$ is defined as :

$$
|\eta|=\sqrt{\eta_{1}^{2}(x)+\eta_{2}^{2}(x)} \quad \forall \eta=\left(\begin{array}{c}
\eta_{1} \\
\eta_{2}
\end{array}\right) .
$$

The variational inequality associated to problem (11) is thus:

$$
\left\{\begin{array}{l}
\text { Find } \eta \in K_{z} \text { such that } \forall \psi \in K_{z} \\
a(\eta, \psi-\eta) \geq l(\psi-\eta)
\end{array}\right.
$$

where $a$ is defined by:

$$
a(\eta, \psi)=\alpha \int_{\Omega} \operatorname{div} \eta \operatorname{div} \psi+\beta \int_{\Omega} \eta \psi,
$$

and $l$ is defined by:

$$
l(\eta)=-\sqrt{\alpha} \int_{\Omega} f \operatorname{div} \eta .
$$

The bilinear form $a$ is symmetrical and coercive in $H_{0}(\operatorname{div}, \Omega)$. Indeed, there exists a constant $c_{\alpha}=\sup (\alpha, \beta)$, such that for all $\eta$ and $\psi \in H_{0}(\operatorname{div}, \Omega)$, one has:

$$
|a(\eta, \psi)| \leq c_{\alpha}\|\eta\|_{H(\operatorname{div}, \Omega)}\|\psi\|_{H(\operatorname{div}, \Omega)},
$$

and there exists a constant $\gamma_{0}=\inf (\alpha, \beta)$ such that for all $\eta \in H_{0}(\operatorname{div}, \Omega)$, one has:

$$
a(\eta, \eta) \geq \gamma_{0}\|\eta\|_{H(\operatorname{div}, \Omega)}^{2} .
$$

\section{A R I M A}


Moreover $l$ is linear and continuous over $H_{0}(\operatorname{div}, \Omega)$ since for all $\eta \in H_{0}(\operatorname{div}, \Omega)$, one has:

$$
|l(\eta)| \leq c_{l}\|\eta\|_{H(\operatorname{div}, \Omega)} .
$$

where $c_{l}=\sqrt{\alpha}\|f\|_{0, \Omega}$.

\section{The interpolation error estimates}

We now focus our interest on the analysis of the approximation error due to the use of quadrilateral finite elements method by discretizing the domain with quadrilateral rectangles. We are most interested in the analysis of the different ways of projection in the restoration algorithm (10), in order to provide the best restoration results.

\subsection{A brief recall on Raviart-Thomas's element [19]}

Let $\Omega$ be a bounded, open subset of $\mathbb{R}^{2}$, having a boundary $\Gamma, \Gamma=\partial \Omega$, which is piecewise $C^{1}$. Let $\mathcal{T}^{h}$ be a decomposition of $\bar{\Omega}$ into compact sets $K$, such that $\bar{\Omega}=$ $\cup_{K \in \mathcal{T}^{h}} K$. The elements $K$ are obtained as the range of a reference elements $\hat{K}$ by the transformation $F_{K}$ (see figure 3) which is a linear and bijective mapping defined by the following relation (see [20]):

$$
x=F(\hat{x})=B \hat{x}+b,
$$

where $B$ is an invertible matrix and $b$ a vector on $\mathbb{R}^{2}$.

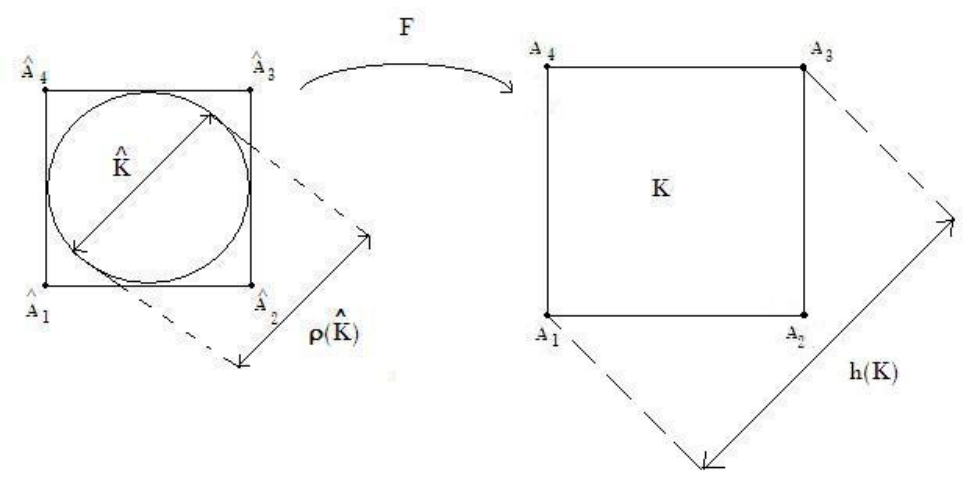

Figure 3. The mapping $F$. 
The derivative of this function is then $D F=B$, and the determinant is $\operatorname{det}(D F)=$ $\operatorname{det} B$.

Let us now introduce the following expression of the norm for a vector $v \in \mathbb{R}^{2}$ :

$$
\|v\|_{p}=\left[\sum_{i=1: 2}\left|v_{i}\right|^{p}\right]^{1 / p},
$$

and the expression of the norm of matrix $B$ :

$$
\|B\|=\sup _{\xi \neq 0} \frac{|B \xi|}{|\xi|}
$$

where $|\xi|$ is the Euclidean norm of vector $\xi$ in $\mathbb{R}^{2}$.

Denoting by $h(K)$ the diameter of $K$, and $\rho(K)$ the maximum diameter of the balls included in it (see figure (3)), we thus have the following result:

Lemma 3.1 ([8]) The following majoration of the norms holds:

$$
\|B\| \leq \frac{h(K)}{\rho(\hat{K})} \quad, \quad\left\|B^{-1}\right\| \leq \frac{h(\hat{K})}{\rho(K)} .
$$

Besides, there exists a constant $\delta$ such that:

$$
|\operatorname{det} B| \leq \delta[h(K)]^{2} .
$$

Let us recall the Hilbert space:

$$
H^{m}(\Omega)=\left\{v \in L^{2}(\Omega), \partial^{\gamma} v \in L^{2}(\Omega),|\gamma| \leq m\right\},
$$

and its semi-norm:

$$
|v|_{m, \Omega}=\left(\sum_{|\gamma|=m}\left\|\partial^{\gamma} v\right\|_{0, \Omega}^{2}\right)^{1 / 2}
$$

as well as the norm on the Hilbert space $H(\operatorname{div}, \Omega)\left(\operatorname{resp} . H_{0}(\operatorname{div}, \Omega)\right)$ :

$$
\|\eta\|_{H(\operatorname{div}, \Omega)}=\left[\|\eta\|_{L^{2}(\Omega)}^{2}+\|\operatorname{div} \eta\|_{L^{2}(\Omega)}^{2}\right]^{\frac{1}{2}} .
$$


According to the decomposition of $\bar{\Omega}$, the Raviart Thomas's finite element used considers the shape vectors $q_{k}$, which are defined on the square element $K$, centered at point $\left(x_{1}^{K}, x_{2}^{K}\right)$, by ( $h$ is the mesh size ie the the side length of the square $K$ ):

$$
\begin{aligned}
& q_{1}^{K}=\left\{\begin{array}{l}
\frac{h+2\left(x_{1}-x_{1}^{K}\right)}{2 h} \\
0
\end{array} q_{2}^{K}=\left\{\begin{array}{l}
0 \\
\frac{h+2\left(x_{2}-x_{2}^{K}\right)}{2 h}
\end{array}\right.\right. \\
& q_{3}^{K}=\left\{\begin{array}{l}
\frac{h-2\left(x_{1}-x_{1}^{K}\right)}{2 h} \\
0
\end{array} q_{4}^{K}=\left\{\begin{array}{l}
0 \\
\frac{h-2\left(x_{2}-x_{2}^{K}\right)}{2 h} .
\end{array}\right.\right.
\end{aligned}
$$

The simplest approximation space for $H_{0}(\operatorname{div}, \Omega)$ is thus defined as it follows:

$$
V^{h}=\left\{\eta\left|\eta \in H_{0}(\operatorname{div}, \Omega), \forall K \in \mathcal{T}^{h}, \eta\right|_{K}=\sum_{k=1,2,3,4} \eta_{k}^{K} q_{k}^{K}\left(x_{1}, x_{2}\right)\right\} .
$$

The interpolation operator $\Pi_{h}$ of $H_{0}(\operatorname{div}, \Omega)$ in $V^{h}$, is thus defined as follows:

$$
\forall \gamma, \gamma \text { side of the mesh } \mathcal{T}^{h}, \int_{\gamma} \Pi_{h} \eta \cdot n_{\gamma}=\int_{\gamma} \eta \cdot n_{\gamma},
$$

where $n_{\gamma}$ is the outing normal vector to $\gamma$.

Theorem 3.1 ([20]) Provided the family mesh $\mathcal{T}_{h}$ is uniformly regular ( $F_{K}$ is linear), there exists a constant $c$ independent on $h$ and $\eta$, such that:

$$
\| \eta-\left.\Pi_{h} \eta\right|_{0, \Omega} \leq c h|\eta|_{1, \Omega}, \quad \forall \eta \in\left[H^{1}(\Omega)\right]^{2},
$$

and if $\operatorname{div}(\eta) \in H^{1}(\Omega)$, then:

$$
\left.|| \operatorname{div}\left(\eta-\Pi_{h} \eta\right)\right|_{0, \Omega} \leq \operatorname{ch}|\operatorname{div}(\eta)|_{1, \Omega} .
$$

Given the previous results, one can now establish the standard following error bound which is given in Raviart-Thomas [19] and Roberts-Thomas [20] for $p=2$.

Theorem 3.2 For all $\eta$ in $H(\operatorname{div}, \Omega) \cap\left[W^{1, p}(\Omega)\right]^{2}$ where $p \geq 2$, there exists a constant $c$ independent on $h$ such that:

$$
\left|\eta-\Pi_{h} \eta\right|_{0, p, \Omega} \leq c h|\eta|_{1, p, \Omega} .
$$




\subsection{Estimates of the interpolation error}

Let us denote by $z_{h}$, the approximation of $z$, on the set $L^{h}$ of piecewise constant functions, which constitute an inner finite dimensional approximation of $L^{2}(\Omega)$ :

$$
L^{h}=\left\{z^{h} \in L^{2}(\Omega) ; z_{\left.\right|_{K}}^{h} \in P_{0}(K), \forall K \in \mathcal{T}^{h}\right\}
$$

then the approximation space of $\mathcal{D}$, denoted by $\mathcal{D}_{h}$, is the intersection of sets $V^{h} \times L^{h}$ and $\mathcal{D}$, and it is defined as:

$$
\mathcal{D}^{h}=\left\{\left(\eta_{h}, z_{h}\right) \in V^{h} \times L^{h} ;\left|\eta_{h}(\chi)\right|^{2}+\left|z_{h}(\chi)\right|^{2} \leq \varepsilon^{2} ; z_{h}(\chi) \geq 0, \forall \chi \in \mathcal{N}\right\},
$$

where $\mathcal{N}$ is the set of the nodes of the mesh which are the center points of the elements $K$.

Let us now define the convex set $K_{z_{h}}$, as:

$$
K_{z_{h}}=\left\{\eta_{h} \in V^{h},\left|\eta_{h}(\chi)\right| \leq \sqrt{\varepsilon^{2}-\left|z_{h}(\chi)\right|^{2}}, \forall \chi \in \mathcal{N}\right\}
$$

Remark 3.1 The convex set $K_{z_{h}}$ is not a subset of the convex set $K_{z}$ defined above in (12):

$$
K_{z}=\left\{\eta \in H_{0}(\operatorname{div}, \Omega),|\eta| \leq \sqrt{\varepsilon^{2}-z^{2}}\right\}
$$

The approximation problem, associated to (13), is then the following one:

$$
\left\{\begin{array}{l}
\text { find } \eta_{h} \in K_{z_{h}} \text { such that } \forall \psi_{h} \in K_{z_{h}}: \\
a\left(\eta_{h}, \psi_{h}-\eta_{h}\right) \geq l\left(\psi_{h}-\eta_{h}\right) .
\end{array}\right.
$$

The solutions $\eta$ of problem (13) and $\eta_{h}$ of the approximation problem (35) satisfy the following results:

Lemma 3.2 There exists a constant $C$ independent on h such that:

$$
\|\eta\|_{H(\operatorname{div}, \Omega)} \leq C\|f\|_{0, \Omega}
$$

and

$$
\left\|\eta_{h}\right\|_{H(\operatorname{div}, \Omega)} \leq C\|f\|_{0, \Omega} .
$$

Proof:

From the variational inequality (35), one deduces:

$$
l\left(\psi_{h}-\eta_{h}\right) \leq a\left(\eta_{h}, \psi_{h}-\eta_{h}\right), \forall \psi_{h} \in K_{z_{h}} .
$$

\section{A R I M A}


By setting $\psi_{h}=0$ in this inequality and by using (18), one obtains:

$$
a\left(\eta_{h}, \eta_{h}\right) \leq l\left(\eta_{h}\right) \leq c_{l}\left\|\eta_{h}\right\|_{H(\operatorname{div}, \Omega)} .
$$

Since $c_{l}=\sqrt{\alpha}\|f\|_{0, \Omega}$ and $a$ is coercive (17), one obtains the following inequality:

$$
\gamma_{0}\left\|\eta_{h}\right\|_{H(\operatorname{div}, \Omega)} \leq \sqrt{\alpha}\|f\|_{0, \Omega}
$$

Then we deduce the result for $C=\sqrt{\alpha} / \gamma_{0}$.

$\eta$ being the solution of problem (11), and assuming $|\Omega| \leq 1$, one can now deduce the following result:

Lemma 3.3 If $|\Omega| \leq 1$ then for $(\eta, z) \in \mathcal{D}$, and $\left(\eta_{h}, z_{h}\right) \in \mathcal{D}_{h}$, there exists a constant $c_{z}$ such that the following result holds:

$$
\left\|z-z_{h}\right\|_{0, \Omega} \leq \sqrt{2}\left(\varepsilon+c_{z} h\right) .
$$

Proof:

We notice that $z \leq \sqrt{\varepsilon^{2}-|\eta|^{2}}$ on $\Omega$ and it exists a constant $c_{z}>0$ such that :

$$
z_{h} \leq \sqrt{\varepsilon^{2}-\left|\Pi_{h} \eta\right|^{2}}+c_{z} h
$$

Then:

$$
\begin{aligned}
\int_{\Omega}\left|z-z_{h}\right|^{2} & \leq \int_{\Omega}|z|^{2}+\left|z_{h}\right|^{2}, \\
& \leq 2\left(\varepsilon+c_{z} h\right)^{2}|\Omega| .
\end{aligned}
$$

Bounding $|\Omega|$ by 1 , lemma (3.3) is proved.

Finally, for $|\Omega| \leq 1$ the following main result concerning the estimation of the error holds for $\eta \in K_{z}$ :

Theorem 3.3 For the family of quadriangulations $\mathcal{T}_{h}$ assumed to be uniformly regular, and by assuming that the parameters $\beta$ and $\alpha$ are lower than 1, there exists a constant $c$ independent on $h$, such that:

$$
\left\|\eta-\eta_{h}\right\|_{H(d i v, \Omega)} \leq c h^{\frac{1}{2}}
$$

\section{Proof:}

For $\eta \in K_{z}$ and $\eta_{h} \in K_{z_{h}}$ and from the variational inequalities (13) and (35), one deduces:

$$
l(\psi-\eta) \leq a(\eta, \psi-\eta), \forall \psi \in K_{z},
$$


72 A R I M A - Volume 15-2012

$$
l\left(\psi_{h}-\eta_{h}\right) \leq a\left(\eta_{h}, \psi_{h}-\eta_{h}\right), \forall \psi_{h} \in K_{z_{h}} .
$$

Then, for all $\psi \in K_{z}$ and $\psi_{h} \in K_{z_{h}}$ :

$$
\begin{aligned}
a\left(\eta-\eta_{h}, \eta-\eta_{h}\right)= & a\left(\eta, \eta-\eta_{h}\right)-a\left(\eta_{h}, \eta-\eta_{h}\right), \\
= & a(\eta, \eta-\psi)+a\left(\eta, \psi-\eta_{h}\right)- \\
& a\left(\eta_{h}, \psi_{h}-\eta_{h}\right)+a\left(\eta_{h}, \psi_{h}-\eta\right), \\
\leq & l(\eta-\psi)+l\left(\eta_{h}-\psi_{h}\right)+a\left(\eta, \psi-\eta_{h}\right)+a\left(\eta_{h}, \psi_{h}-\eta\right) .
\end{aligned}
$$

Since the bilinear form (14) and the linear operator defined in (15) are both continuous:

$$
\begin{aligned}
& a\left(\eta-\eta_{h}, \eta-\eta_{h}\right) \leq \sqrt{\alpha}\|f\|_{0, \Omega}\left[\left\|\eta-\psi_{h}\right\|_{H(\operatorname{div}, \Omega)}+\left\|\psi-\eta_{h}\right\|_{H(\operatorname{div}, \Omega)}\right]+ \\
& c_{\alpha}\|\eta\|_{H(\operatorname{div}, \Omega)}\left\|\psi-\eta_{h}\right\|_{H(\operatorname{div}, \Omega)}+c_{\alpha}\left\|\eta_{h}\right\|_{H(\operatorname{div}, \Omega)}\left\|\psi_{h}-\eta\right\|_{H(\operatorname{div}, \Omega)} .
\end{aligned}
$$

From the coerciveness of $a(17)$, one gets:

$$
\begin{aligned}
\gamma_{0}\left\|\eta-\eta_{h}\right\|_{H(\operatorname{div}, \Omega)}^{2} & \leq\left[\sqrt{\alpha}\|f\|_{0, \Omega}+c_{\alpha}\left\|\eta_{h}\right\|_{H(\operatorname{div}, \Omega)}\right]\left\|\eta-\psi_{h}\right\|_{H(\operatorname{div}, \Omega)}+ \\
& {\left[\sqrt{\alpha}\|f\|_{0, \Omega}+c_{\alpha}\|\eta\|_{H(\operatorname{div}, \Omega)}\right]\left\|\eta_{h}-\psi\right\|_{H(\operatorname{div}, \Omega)} }
\end{aligned}
$$

and from the majorations (36) and (37) we deduce:

$$
\begin{aligned}
\gamma_{0}\left\|\eta-\eta_{h}\right\|_{H(\operatorname{div}, \Omega)}^{2} & \leq\left[\sqrt{\alpha}\|f\|_{0, \Omega}+c_{\alpha} C\|f\|_{0, \Omega}\right]\left\|\eta-\psi_{h}\right\|_{H(\operatorname{div}, \Omega)}+ \\
& {\left[\sqrt{\alpha}\|f\|_{0, \Omega}+c_{\alpha} C\|f\|_{0, \Omega}\right]\left\|\eta_{h}-\psi\right\|_{H(\operatorname{div}, \Omega)} . }
\end{aligned}
$$

Setting $A=\frac{1}{\gamma_{0}}\left(\sqrt{\alpha}\|f\|_{0, \Omega}+c_{\alpha} C\|f\|_{0, \Omega}\right)$, one fianlly obtains:

$$
\left\|\eta-\eta_{h}\right\|_{H(\operatorname{div}, \Omega)}^{2} \leq A\left(\left\|\eta-\psi_{h}\right\|_{H(\operatorname{div}, \Omega)}+\left\|\eta_{h}-\psi\right\|_{H(\operatorname{div}, \Omega)}\right)
$$

and eventually:

$\left\|\eta-\eta_{h}\right\|_{H(\operatorname{div}, \Omega)}^{2} \leq A\left(\inf _{\psi_{h} \in K_{z_{h}}}\left\|\eta-\psi_{h}\right\|_{H(\operatorname{div}, \Omega)}+\inf _{\psi \in K_{z}}\left\|\eta_{h}-\psi\right\|_{H(\operatorname{div}, \Omega)}\right)$.

Step 1 : Determining an upper bound to $\inf _{\psi_{h} \in K_{z_{h}}}\left\|\eta-\psi_{h}\right\|_{H(\operatorname{div}, \Omega)}$ :

Let us set $\psi_{h} \in V^{h}$, such that:

$$
\psi_{h}(\chi)=\frac{\sqrt{\varepsilon^{2}-z_{h}^{2}(\chi)}}{\sqrt{\varepsilon^{2}-z^{2}(\chi)}} \eta(\chi), \forall \chi \in \mathcal{N}
$$

\section{A R I M A}


Since $\eta \in K_{z}$, it follows that $\psi_{h} \in K_{z_{h}}$.

The expression of $\psi_{h}$ implies, that it exists a constant $c_{1}>0$ independent on $h$, such that:

$$
\left|\psi_{h}(x)-\eta(x)\right| \leq\left|\sqrt{\varepsilon^{2}-z_{h}^{2}(x)}-\sqrt{\varepsilon^{2}-z^{2}(x)}\right|+c_{1} h, \forall x \in \Omega .
$$

In order to bound from above the second term of this inequality let us use the following property for an arbitrary function $f$ :

$$
|f(x)-f(y)| \leq \int_{y}^{x}\left|f^{\prime}(t)\right| d t
$$

By setting $f(t)=\sqrt{\varepsilon^{2}-t^{2}}$, one has:

$$
\left|\sqrt{\varepsilon^{2}-z_{h}^{2}}-\sqrt{\varepsilon^{2}-z^{2}}\right| \leq \int_{z}^{z_{h}} \frac{|t|}{\sqrt{\varepsilon^{2}-t^{2}}} d t .
$$

From Hölder's inequality, one can claim that for all $p$ and $q$ such that $\frac{1}{p}+\frac{1}{q}=1$ :

$$
\left|\sqrt{\varepsilon^{2}-z_{h}^{2}}-\sqrt{\varepsilon^{2}-z^{2}}\right| \leq\left(\int_{z}^{z_{h}}|t|^{p} d t\right)^{1 / p}\left(\int_{z}^{z_{h}} \frac{1}{\left(\varepsilon^{2}-t^{2}\right)^{q / 2}} d t\right)^{1 / q} .
$$

Setting $s=\varepsilon-t$, one has $\varepsilon^{2}-t^{2}=-s^{2}+2 \varepsilon s$, and:

$$
\frac{1}{\left(\varepsilon^{2}-t^{2}\right)^{q / 2}} \simeq \frac{1}{(2 \varepsilon s)^{q / 2}}=\frac{1}{(2 \varepsilon)^{q / 2}} \cdot \frac{1}{(s)^{q / 2}} .
$$

Thus the term in (56) can be integrate provided $\frac{q}{2}<1$, which means that for all $q$, $1<q<2$ there exists a constant $c_{q}$ such that:

$$
\left(\int_{z}^{z_{h}} \frac{1}{\left(\varepsilon^{2}-t^{2}\right)^{q / 2}} d t\right)^{1 / q} \leq c_{q}
$$

and therefore for all $1<q<2$, (which means that for all $p>2$ ):

$$
\left|\sqrt{\varepsilon^{2}-z_{h}^{2}}-\sqrt{\varepsilon^{2}-z^{2}}\right| \leq c_{q}\left(\int_{z}^{z_{h}}\left|t^{p}\right| d t\right)^{1 / p} .
$$

Finally:

$$
\begin{aligned}
\left(\int_{z}^{z_{h}} t^{p} d t\right)^{1 / p} & \leq\left(\left[\frac{\left|t^{p+1}\right|}{p+1}\right]_{z}^{z_{h}}\right)^{1 / p} \\
& \leq c_{p}\left|z_{h}^{p+1}-z^{p+1}\right|^{1 / p}
\end{aligned}
$$


with $c_{p}=\left(\frac{1}{p+1}\right)^{1 / p}$.

In order to find an upper bound to the second term of the inequality (59), let us notice that for any $f$ continuously differentiable, one has:

$$
|f(x)-f(y)| \leq \sup _{t \in \mathbb{R}}\left|f^{\prime}(t)\right| \cdot|x-y| .
$$

Setting $f(t)=t^{p+1}$, one obtains that for all $t$ (such that $t \in\left[z, z_{h}\right]$ if $z_{h} \geq z$, and $t \in\left[z_{h}, z\right]$ otherwise), the following result holds:

$$
\left|f^{\prime}(t)\right| \leq(p+1) \varepsilon^{p} .
$$

Replacing the function $f$, defined at (60), one has:

$$
\left|z_{h}^{p+1}(x)-z^{p+1}(x)\right| \leq(p+1) \varepsilon^{p}\left|z_{h}(x)-z(x)\right|, \forall x \in \Omega .
$$

From inequalities (58) and (59), there exists a constant $c_{\varepsilon}=c_{q} \cdot \varepsilon$ such that for all $p>2$ :

$$
\left|\sqrt{\varepsilon^{2}-z_{h}^{2}(x)}-\sqrt{\varepsilon^{2}-z^{2}(x)}\right| \leq c_{\varepsilon}\left|z_{h}(x)-z(x)\right|^{1 / p} .
$$

Using the $L^{2}$ norm for (53), one obtains that it exists a constant $c_{2}$ such that:

$$
\left\|\psi_{h}-\eta\right\|_{0, \Omega} \leq \sqrt{2}\left\|\sqrt{\varepsilon^{2}-z_{h}^{2}}-\sqrt{\varepsilon^{2}-z^{2}}\right\|_{0, \Omega}+c_{2} h,
$$

and from the upper bound (62):

$$
\left\|\psi_{h}-\eta\right\|_{0, \Omega} \leq \sqrt{2} c_{\varepsilon}\left\|\left(z_{h}-z\right)^{1 / p}\right\|_{0, \Omega}+c_{2} h .
$$

Since for $p^{\prime}>2$ the inclusion $L^{p^{\prime}}(\Omega) \subset L^{2}(\Omega)$ is satisfied, the $L^{2}$ norm can be replaced by the $L^{p^{\prime}}$ norm; therefore:

$$
\left\|\psi_{h}-\eta\right\|_{0, \Omega} \leq \sqrt{2} c_{\varepsilon}\left\|\left(z_{h}-z\right)^{1 / p}\right\|_{L^{p^{\prime}}(\Omega)}+c_{2} h .
$$

Setting $p^{\prime}=2 p$ with $p>2$, it comes out that:

$$
\begin{aligned}
\left\|\psi_{h}-\eta\right\|_{0, \Omega} & \leq \sqrt{2} c_{\varepsilon}\left[\int_{\Omega}\left(z_{h}-z\right)^{2}\right]^{\frac{1}{2 p}}+c_{2} h, \\
& \leq c_{\varepsilon}\left\|z_{h}-z\right\|_{0, \Omega}^{\frac{1}{p}}+c_{2} h
\end{aligned}
$$

and with the estimation (41), one deduces that:

$$
\left\|\psi_{h}-\eta\right\|_{0, \Omega} \leq 2 c_{\varepsilon}\left(\varepsilon+c_{z} h\right)^{\frac{1}{p}}+c_{2} h .
$$


From a similar argument, the divergence operator also satisfies:

$$
\left\|\operatorname{div}\left(\psi_{h}-\eta\right)\right\|_{0, \Omega} \leq 2 c_{\varepsilon}\left(\varepsilon+c_{z} h\right)^{\frac{1}{p}}+c_{2} h .
$$

Finally, one deduces that there exists a constant $c^{\prime}$ such that the following result is true:

$$
\inf _{\psi_{h} \in K_{z_{h}}}\left\|\eta-\psi_{h}\right\|_{H(\operatorname{div}, \Omega)} \leq\left\|\eta-\psi_{h}\right\|_{H(\operatorname{div}, \Omega)} \leq c^{\prime} h .
$$

Step 2: Finding an upper bound to $\inf _{\psi \in K_{z}}\left\|\eta_{h}-\psi\right\|_{H(\operatorname{div}, \Omega)}$ :

Since $\eta_{h} \in K_{z_{h}}$, one derives the upper bound:

$$
\left|\eta_{h}(\chi)\right| \leq \sqrt{\varepsilon^{2}-z_{h}^{2}(\chi)}, \forall \chi \in \mathcal{N} .
$$

From an extension of this upper bound over all the domain $\Omega$, one can claim that there exists a constant $c_{3}>0$ independent on $h$, such that:

$$
\left|\eta_{h}(x)\right| \leq \sqrt{\varepsilon^{2}-z_{h}^{2}(x)}+c_{3} h, \forall x \in \Omega .
$$

Let us set:

$$
\psi=\frac{\sqrt{\varepsilon^{2}-z^{2}}}{\sqrt{\varepsilon^{2}-z_{h}^{2}}+c_{3} h} \eta_{h} .
$$

Then:

$$
|\psi(x)|=\frac{\sqrt{\varepsilon^{2}-z^{2}(x)}}{\sqrt{\varepsilon^{2}-z_{h}^{2}(x)}+c_{3} h}\left|\eta_{h}(x)\right|, \forall x \in \Omega .
$$

Therefore:

$$
|\psi(x)| \leq \sqrt{\varepsilon^{2}-z^{2}(x)}, \forall x \in \Omega
$$

which leads to: $\psi \in K_{z}$.

From the expression of $\psi$ and the majoration (70), one has the following inequality:

$$
\left|\psi(x)-\eta_{h}(x)\right| \leq\left|\sqrt{\varepsilon^{2}-z^{2}(x)}-\sqrt{\varepsilon^{2}-z_{h}^{2}(x)}\right|+c_{3} h, \forall x \in \Omega .
$$

The inequality (62) is proved and by using the same arguments for the divergence operator one concludes that there exists a constant $c^{\prime \prime}$ independent on $h$, such that one has the following result:

$$
\inf _{\psi \in K_{z}}\left\|\eta_{h}-\psi\right\|_{H(\operatorname{div}, \Omega)} \leq\left\|\eta_{h}-\psi\right\|_{H(\operatorname{div}, \Omega)} \leq c^{\prime \prime} h .
$$

Then by the inequalities (69) and (75) and the majoration (51), one deduces that there exists a constant $c(f, \varepsilon, \alpha, \beta)=\sqrt{A\left(c^{\prime}+c^{\prime \prime}\right)}$, verifying the following inequality:

$$
\left\|\eta-\eta_{h}\right\|_{H(\operatorname{div}, \Omega)} \leq c(f, \varepsilon, a, \beta) h^{\frac{1}{2}}
$$




\section{Numerical results}

In this section, we compare the restoration results obtained with the projection algorithm (10) using the projection on the interior edges midpoints of the elements $K$, and the one using the projection on the center points of the elements $K$. This comparison will be made by calculating the interpolation error and also the SNR coefficient, which is the parameter commonly used to assess the restoration results.

We recall that the SNR is the signal-to-noise ratio, used to estimate the quality of an image $I_{2}$ with respect to a reference image $I_{1}$, defined by the expression:

$$
S N R\left(I_{1} / I_{2}\right)=10 \log _{10}\left[\frac{\sigma^{2}\left(I_{1}\right)}{\sigma^{2}\left(I_{1}-I_{2}\right)}\right]
$$

where $\sigma$ is the variance.

We begin by presenting a 2D processed image, obtained using the dual algorithm with the choice of parameters $\left(\varepsilon=1, \alpha=0.07\right.$ and $\beta=10^{-3}$ ) (which guarantees the best restoration results).

Figure 4 displays the original image which is a squared image 115 pixels $\times 115$ pixels, and the associated noisy image $f$ obtained by adding a Gaussian noise, whereas Figure 5 shows the restored images obtained by using the dual algorithm. In Figure 5, image (a) is obtained by using the projection on the interior edges midpoints of the elements $K$ whereas image (b) is obtained using the projection on the center points of the elements $K$.

To compare these two restoration results, we compute the approximation error of $\eta$. One can observe from figure 6 that the projection on the center points of the elements $K$ (which is the method proposed and studied in this paper) provides a better restored image than the projection on the interior edges midpoints of the elements $K$ (which is a non conforming method).

We can also compare these two restoration results using the coefficient SNR. Then for figure 5(a) the SNR value is $20 \mathrm{~dB}$, whereas its value for figure (reffig:debruit(b) the value of the SNR is $23.63 \mathrm{~dB}$. This confirms the superiority of the proposed projection method (projection on the center points of the elements $K$ ).

Finally, the two methods have been applied to the real (and famous) Lena image. The figure 7 represents the original image figure 7 (a) and the noisy one figure 7 (b). The

\section{A R I M A}


results obtained by the two of them are represented in Figure 8: part (a) for the non conforming method (projection on the interior edges midpoints of the elements $K$ ) and part (b) for the here proposed one (projection on the center points of the elements $K$ ). Once again, one can observe better results for the latter method. Indeed, for Figure 8(a) the norm $\left\|\eta_{h}-\eta\right\|_{H(\operatorname{div}, \Omega)}$ is approximatively $10^{-2}$ (and the SNR is $17.69 \mathrm{~dB}$ ) whereas for Figure 8(b), the norm is approximatively $3 \cdot 10^{-3}$ (and the SNR $20.11 \mathrm{~dB}$ ).

\section{Conclusion}

It has been shown in this paper that one can adapt the Raviart-Thomas's mixed finite element for solving a non linear model including a local projection. The difficulty was to overcome the fact that the local projection induces a non conforming field with respect to the space $H(d i v, \Omega)$. But a local averaging of the normal flux at the middle nodes of each side of the mesh (there are two values at these points) enables one to stabilize the method and leads to improved numerical results. This method is very convenient in particular for image processing, where non linear operators are required for denoising and preserving the boundary of the objects.

\section{Bibliography}

[1] G. Acosta and R. G. Duran, The maximum angle condition for mixed and non conforming elements: Application to the Stokes equations, SIAM J. Numer. Anal. 37 (2000), 18-36.

[2] T. Apel, Anisotropic finite elements: Local estimates and applications, Series Advances in Numerical Mathematics, Teubner, Stuttgart (1999).

[3] G. Aubert and P. Kornprobst,Mathematical problems in image processing, SpringerVerlag, New-York (2002).

[4] I. Babuska and A. K. Aziz, On the angle condition in the finite element method, SIAM J. Numer. Anal., 13 (1976), 214-226.

[5] S. Brenner and L. R. Scott, The Mathematical Analysis of Finite Element Methods, Springer-Verlag (1994).

[6] A. Chambolle, An algorithm for total variation minimization and applications, $J$. Math. Imaging and Vision, 20 (2004), 89-97.

[7] P. Charbonnier, Reconstruction d'Image, Régularisation avec Prise en Compte des Discontinuités, Ph. D. Thesis. Université de Nice-Sophia Antipolis (1994).

[8] P.G. Ciarlet, Basic Error Estimates for Elliptic Problems, Handbook of Numerical Analysis, Vol II, Finite Element methods (Part 1), Elsevier, Amsterdam (1991). 
[9] P.G. Ciarlet, Introduction à l'analyse numérique matricielle et à l'optimisation, Masson, Paris (1994)

[10] R. Deriche and O. Faugeras, Les EDP en traitement des images et vision par ordinateur, INRIA research report 2497 (1994).

[11] Ph. Destuynder, Analyse et traitement des images numériques, Hermès, Paris (2006).

[12] Ph. Destuynder, M. Jaoua and H. Sellami, A dual algorithm for denoising and preserving edges in image processing, International Journal of Inverse and Ill-Posed Problems, 15 (2007), 149-165.

[13] R. G. Duran, Error estimates for 3-d narrow finite elements, Math. Comp., 68 (1999), 187-199.

[14] R. G. Duran, Error estimates for anisotropic finite elements and applications, Proceedings of the International Congress of Mathematicians (2006).

[15] R. G. Duran and A. L. Lombardi, Error estimates on anisotropic Q1 elements for functions in weighted Sobolev spaces, Math. Comp., 74 (2005), 1679-1706.

[16] H.W. Engl, M. Hanke and A. Neubauer, Regularization of Inverse Problems, Kluwer, Dordrecht (1994).

[17] R. Deriche and O. Faugeras, Les EDP en traitement des images et vision par ordinateur, INRIA research report 2697 (1995).

[18] S. Osher, L. Rudin and E. Fatemi, Non linear total variation based noise removal algorithms, Phys. D, 60 (1992), 259-268.

[19] P. A. Raviart and J. M. Thomas, A mixed finite element method for second order elliptic problems, Mathematical Aspects of the Finite Element Method, (I. Galligani, E. Magenes, eds.), Lectures Notes in Math. 606, Springer-Verlag (1977).

[20] J. E. Roberts and J.-M. Thomas, Mixed and Hybrid Methods, Handbook of Numerical Analysis, Vol. II, Elsevier, Amsterdam (1991). 

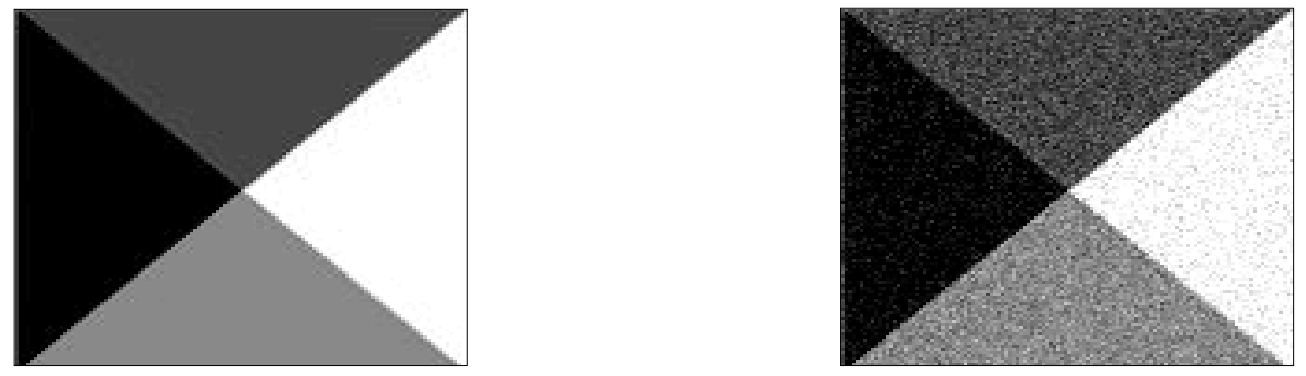

Figure 4. Original (left) and noisy (right) images.

(a)

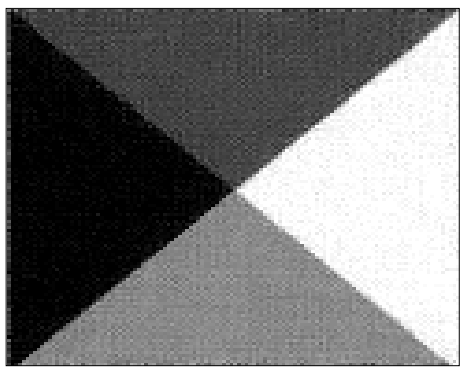

(b)

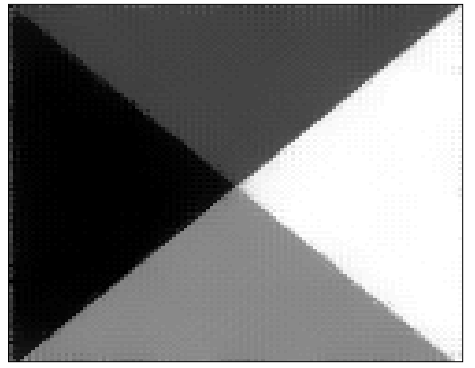

Figure 5. Denoising by different projection methods (left the projection on the interior edges midpoints of the elements $K$ and right the projection on the center points of the elements $K$. 
80 A R I M A - Volume 15-2012

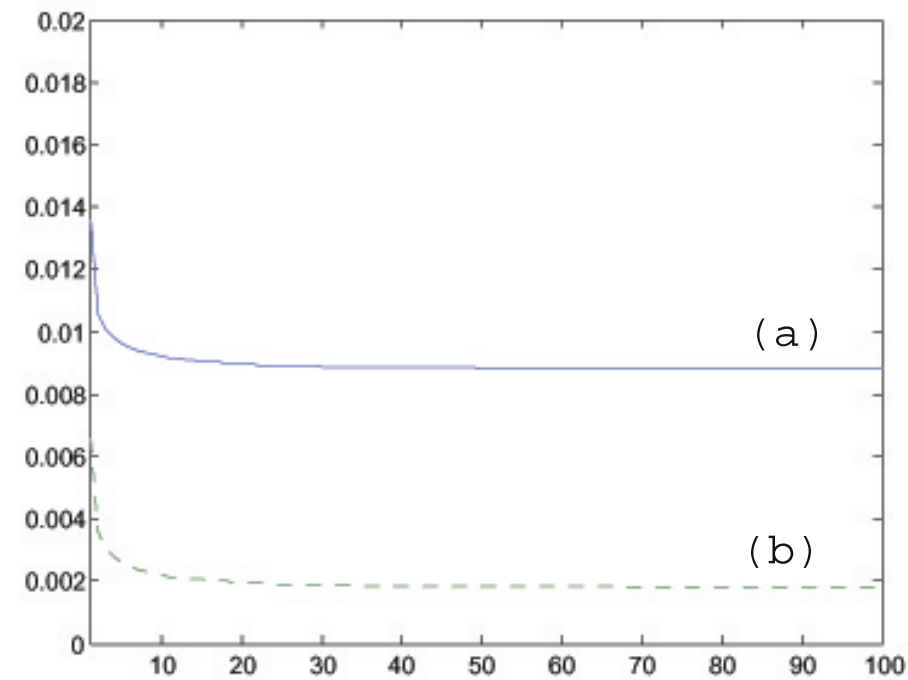

Figure 6. The value of the norm $\left\|\eta_{h}-\eta\right\|_{H(\operatorname{div}, \Omega)}$ as a function of the iteration $k$ of the projected descent algorithm (10), for the two projections: (a) for the non conforming one (the projection on the interior edges midpoints of the elements $K$ ) and (b) for the one suggested here (the projection on the center points of the elements $K$ ).

\section{A R I M A}



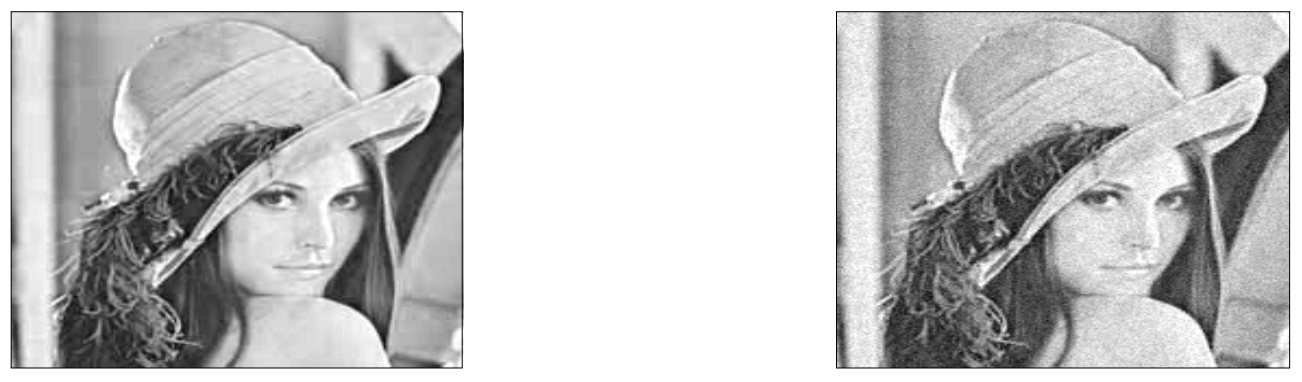

Figure 7. The original and the noisy images (Gaussian noise).

(a)

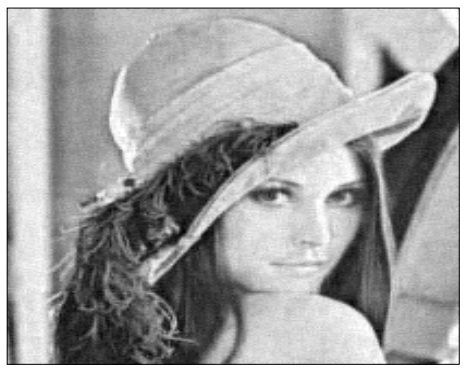

(b)

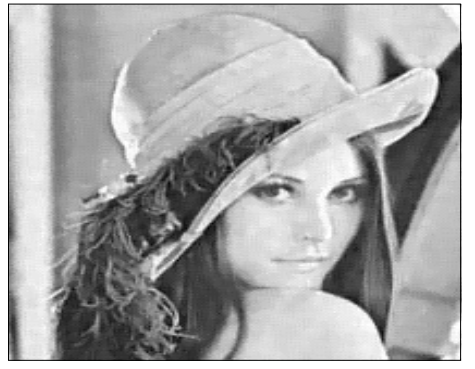

Figure 8. The results of the two projection methods: left the non conforming and right the one suggested here. 